\title{
Disparities in patient enrollment on glioblastoma clinical trials
}

\author{
Yang Liu ${ }^{1}$, Andrea Wasilewski ${ }^{1}$ \& Nimish A Mohile*,1 \\ ${ }^{1}$ Department of Neurosurgery, Department of Neurology, University of Rochester Medical Center, NY 14618, United States \\ *Author for correspondence: Nimish_Mohile@URMC.Rochester.edu
}

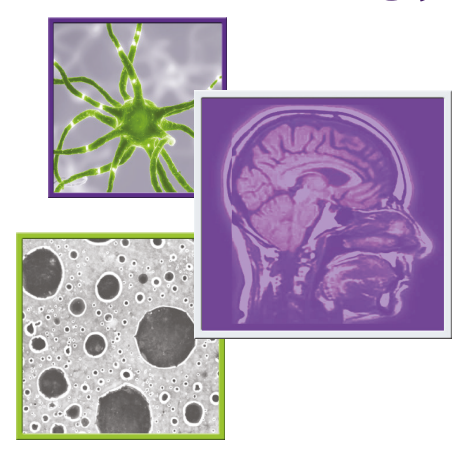

\begin{abstract}
Aim: To determine if enrollment on glioblastoma (GBM) interventional clinical trials (ICTs) in the USA is representative of the population. Materials \& methods: We queried ClinicalTrials.gov for all ICTs in GBM from 1994 to 2019. Demographics were obtained from ClinicalTrials.gov or the trial publication and compared with population data from Central Brain Tumor Registry of the United States. Results: In total, 10617 GBM patients were enrolled in 118 adult ICTs: median age was 54.0 (10.05 years younger than Central Brain Tumor Registry of the United States). Age was most discrepant in recurrent tumors, nonrandomized trials and consortium studies. Median age improved from 52.0 to 59.5 over 25 years. Women represented $37.5 \%$ of subjects. Conclusion: GBM ICTs under-represent older patients but representation of women reflects the population. ICTs need to be designed to better represent the population.
\end{abstract}

First draft submitted: 31 March 2020; Accepted for publication: 12 May 2020; Published online: 30 June 2020

Keywords: age and sex disparities $\bullet$ clinical trials $\bullet$ glioblastoma $\bullet$ trends

Glioblastoma (GBM) is the most common malignant brain tumor in adults and is incurable. Survival of patients on GBM clinical trials has increased over the past decade and ranges from 14 to 20 months in randomized studies [13]. The current standard of care treatment for newly diagnosed GBM consists of radiation with concurrent and adjuvant temzolomide and is based on a randomized clinical trial (RCT), published in 2005, in which the median age was 56 years and patients older than 70 were excluded [1]. More recently, tumor treating fields therapy delivered by Optune ${ }^{\circledR}$ (Novocure, St Helier, Jersey) has been approved as a novel therapeutic approach to GBM and has been included in the National Comprehensive Cancer Network as part of standard of care treatment paradigm. Similarly, the median age was 56 years for this trial. Many cancer clinical trials have preferentially recruited younger patients and results have been extrapolated to older patients [4,5]. This was the case for older patients with GBM until several studies in the past decade have focused on this population. These studies suggest that the median survival of older patients in clinical trials, while markedly shorter than that of younger patients, has increased to a range of 7-9 months [6]. However, examination of population databases reveals that these survival benefits have not been realized across the US population [7]. This may be due to limited evidence-based data to guide clinical care for older patients with GBM, undertreatment of GBM in the community but also due to inadequate representation of a real world population in GBM clinical trials [8,9].

Many studies have demonstrated enrollment disparities with under-representation of older patients, women and racial/ethnic minorities on clinical trials for solid tumors such as lung, breast and colorectal cancer [10-14]. This has been largely unexplored in neuro-oncology and we sought to determine if there were age, sex or racial disparities in patient enrollment in interventional GBM trials using the ClinicalTrials.gov database. We analyzed factors associated with disparities and reported trends in enrollment from 1994 to 2019.

\section{Materials \& methods}

No institutional review board approval was required from our institution (University of Rochester, NY, USA). The ClinicalTrials.gov was queried on 06/18/19 using the following criteria: GBM; USA; completed; 18-64 and older adults (65+); interventional; Phase I, II, III. This yielded 408 studies (Figure 1). Age (median) and sex information were identified from study results posted on ClinicalTrials.gov or relevant publications in PubMed. Trials without median age or sex information were excluded. We calculated the difference in median age (DMA) 


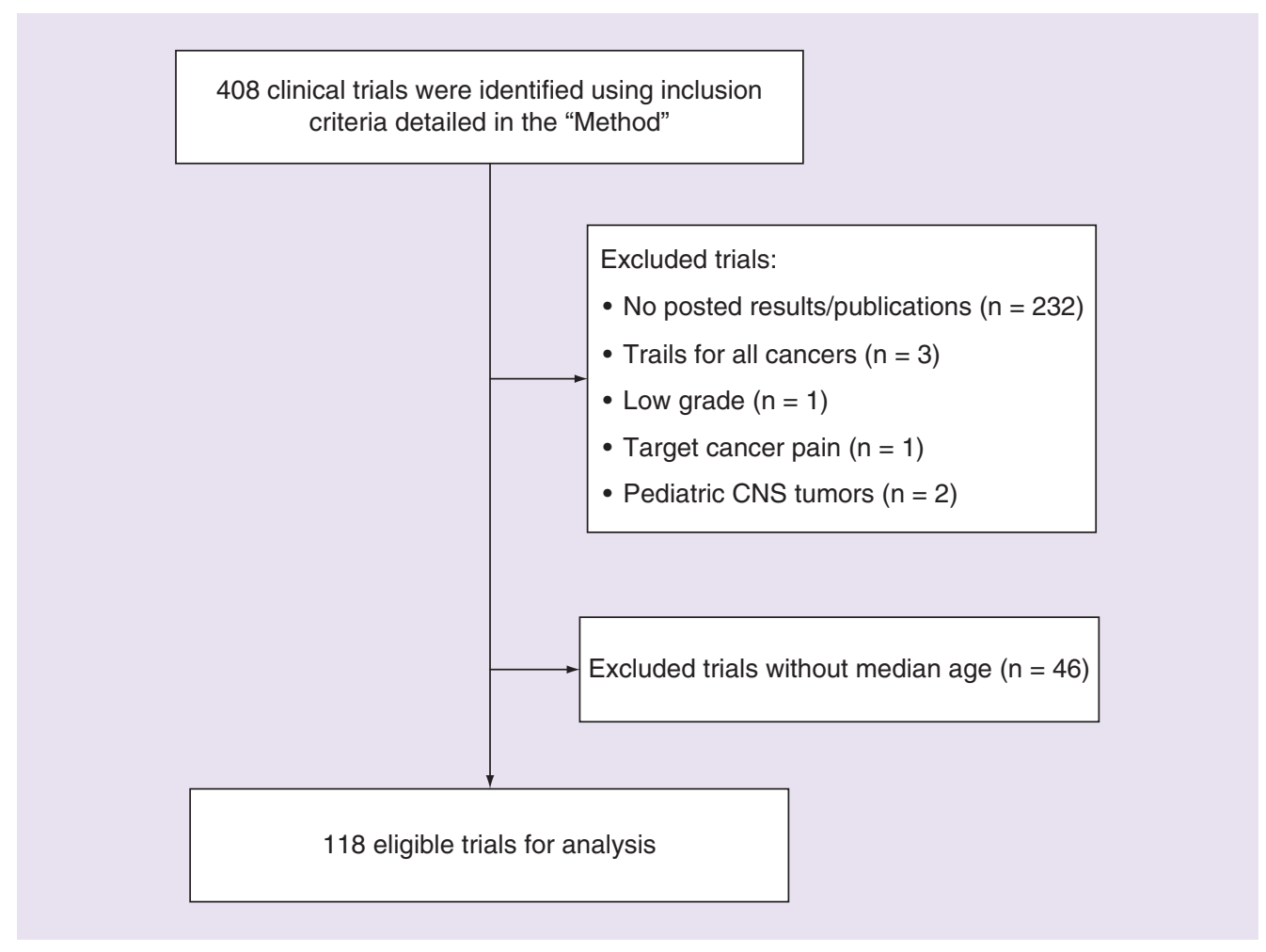

Figure 1. Flowchart of clinical trials identification for the current study. Study inclusion criteria was detailed in the 'Method'.

and the difference in proportion of female patients (DPF) as described by Ludmir EB et al:: DMA was the trial median age minus GBM population median age [13], DPF was the trial proportion of female patients minus GBM proportion of female patients [14]. GBM population data were from the Central Brain Tumor Registry of the United States (CBTRUS) $[7,15,16]$. Funding source of each study was defined as previously described, in other words, a study was classified as industry funded if the sponsor was from industry or a collaborator was from industry [17].

We classified the type of therapeutic intervention based on the National Cancer Institute's (NCI's) definitions on types of cancer treatment; this includes surgery, radiation, chemotherapy, immunotherapy, hormone therapy, stem cell transplant, targeted therapy and precision medicine [18]. For drug interventions, we entered drug names into the NCI Drug Dictionary to determine mechanism of action or postulated mechanism of action and assigned them to a category. Two investigators reviewed these separately and agreed on classification. Drugs that had a mechanism of action intended to enhance the effects of radiation were classified under radiation. The Tumor Treating Fields (Optune ${ }^{\circledR}$, St Helier, Jersey) did not fall into a particular category and we created an additional category that we termed tumor-treatment fields. Investigational agents for which the mechanism of action was unknown or not found in the NCI dictionary were classified as other.

To study trends over time for age and sex disparities, we divided the study period (1994-2019) into three equal 8-year time periods, by year of enrollment initiation: 1994-2002 (group one), 2003-2010 (group two) and 2011-2019 (group three). We compared median age and sex ratio of enrollees to their population data in their respective time period, as described above.

Statistical analyses included independent samples Mann-Whitney U and Kruskal Walls tests, one-sample $t$ test and one-sample Wilcoxon signed rank test as described by Ludmir EB et al. [13,14]. Additionally, one-way ANOVA was used to compare DMA and DPF changes among time intervals. All analyses were conducted with IBM SPSS statistics (version 24). All p-values were two-sided and considered statistically significant when $\mathrm{p}<0.05$.

\section{Results}

118 interventional clinical trials (ICTs) with reported median age and sex information on ClinicalTrials.gov or publications in PubMed were included for further analyses (Figure 1). As shown in Table 1, there were 10,617 


\begin{tabular}{|c|c|c|c|c|}
\hline & 1994-2002 & 2003-2010 & 2011-2019 & Total \\
\hline Number of trials & 33 & 67 & 18 & 118 \\
\hline Age (years, median, $95 \% \mathrm{Cl}$ ) & $52.0(50.0,54.0)$ & $54.0(53.7,55.9)$ & $59.5(56.0,61.5)$ & $54(53.0,55.0)$ \\
\hline $\begin{array}{l}\text { Patient population age } \\
\text { (median, years) }\end{array}$ & 63 & 64 & 65 & NA \\
\hline -Female & $650(37.1 \%)$ & $2852(37.8 \%)$ & $484(36.8 \%)$ & 3986 (37.5\%) \\
\hline $\begin{array}{l}\text { Patient population (female: } \\
\text { male) }\end{array}$ & 0.388 & 0.388 & 0.388 & NA \\
\hline
\end{tabular}

\begin{tabular}{|c|c|c|c|}
\hline Factors & Number of trials & Mean DMA $(95 \% \mathrm{Cl})$ (years) & p-value \\
\hline All included trials & 118 & $-10.05(-10.97,-9.13)$ & $<0.001$ \\
\hline -Newly GBM & 39 & $-7.57(-8.45,-6.62)$ & \\
\hline -Recurrent GBM & 78 & $-11.29(-12.53,-10.13)$ & $<0.001$ \\
\hline \multicolumn{4}{|l|}{ Phase } \\
\hline -Non-III & 109 & $-10.25(-11.21,-9.3)$ & 0.056 \\
\hline \multicolumn{4}{|l|}{ RCT } \\
\hline -Yes & 20 & $-7.65(-8.39,-6.82)$ & \\
\hline$-\mathrm{No}$ & 98 & $-10.54(-11.66,-9.5)$ & 0.004 \\
\hline \multicolumn{4}{|l|}{ Industry } \\
\hline -Yes & 24 & $-7.83(-8.84,-6.83)$ & \\
\hline$-\mathrm{No}$ & 94 & $-10.61(-11.73,-9.69)$ & 0.005 \\
\hline \multicolumn{4}{|l|}{ Modality } \\
\hline -Systemic therapy & 99 & $-10.49(-11.50,-9.48)$ & \\
\hline -Radiation therapy & 6 & $-5.42(-9.49,-1.35)$ & 0.055 \\
\hline -Combination therapy & 9 & $-8.90(-11.59,-6.21)$ & \\
\hline -Others & 4 & $-8.68(-17.05,-0.30)$ & \\
\hline
\end{tabular}

DMA: Difference in median age; GBM: Glioblastoma; RCT: Randomized clinical trial.

patients with GBM enrolled in these ICTs from 1994 to 2019. The median age for GBM clinical trial participants was 54.0 years $(95 \%$ CI: $53.0,55.0)$ while the median age for the GBM population was 63.9 years (95\% CI: 63.9, 64.0) resulting in a difference of 9.9 years and a mean DMA of -10.05 years $(95 \%$ CI: $-10.97,-9.13)$. The median age of the trial population was significantly lower than the GBM population $(\mathrm{p}<0.001)$ (Table 2). Age disparities were more pronounced in recurrent GBM patients (-11.29 years, 95\% CI: -12.53, -10.13) than newly diagnosed GBM patients $(-7.57$ years, $95 \%$ CI: $-8.45,-6.62, \mathrm{p}<0.001)$. Similarly, age disparities were significantly heightened in non-RCT (-10.54 years, $95 \%$ CI: $-11.66,-9.50)$ compared with RCT ( -7.65 years, $95 \%$ CI: $-8.39,-6.82, \mathrm{p}=0.004)$, and noncooperative group trial $(-10.61$ years, $95 \% \mathrm{CI}:-11.73,-9.69)$ compared with cooperative group trial $(-7.83$ years, $95 \% \mathrm{CI}:-8.84,-6.83, \mathrm{p}=0.005)$ (Table 2). Additionally, patients enrolled in early phase clinical trials had greater age disparities compared with those in Phase III trials $(-10.25$ years vs -7.63 years. $\mathrm{p}=0.056)$ as well as patients enrolled in systemic therapy trials compared with radiation therapy $(-10.49$ years vs -5.42 years, $\mathrm{p}=0.055)($ Table 2$)$. 


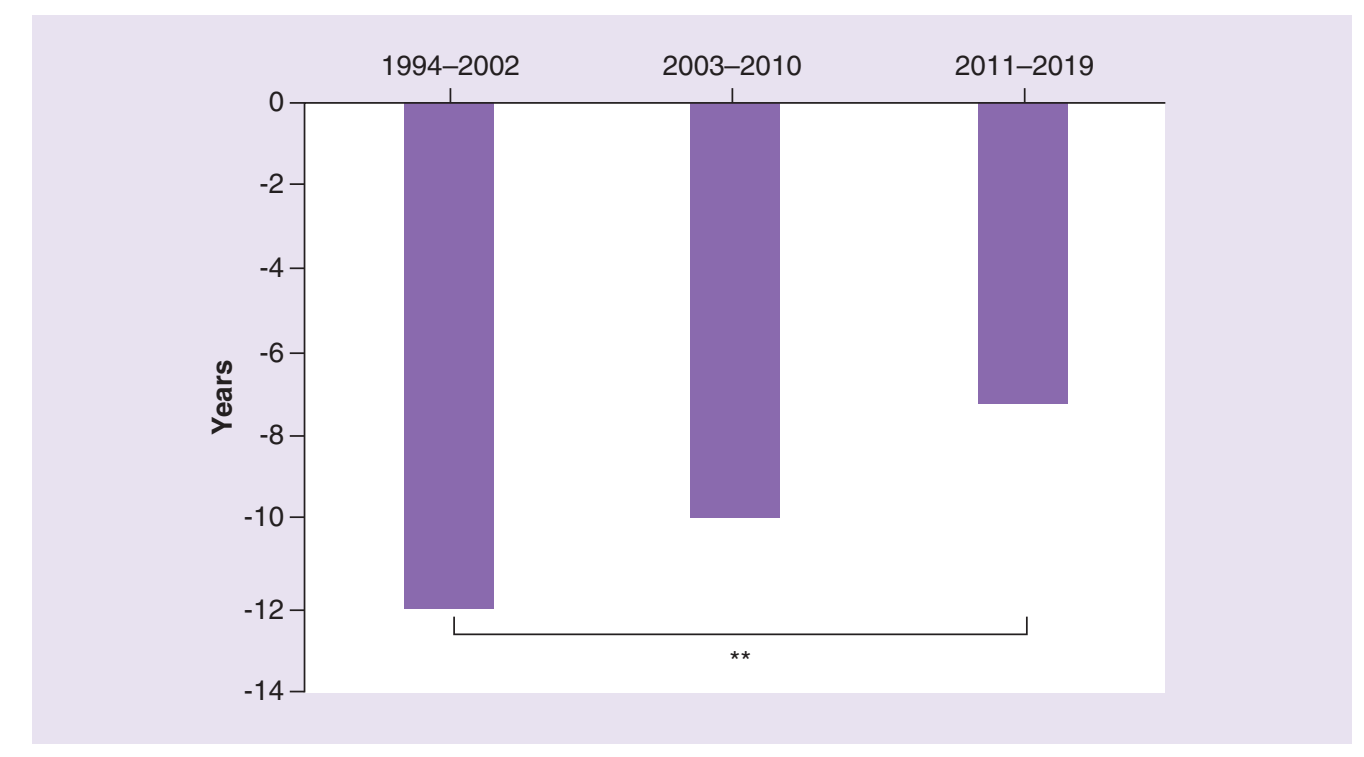

Figure 2. Time changes of difference in median age.

$* * p<0.01$.

Next, we studied time trends and found that age discrepancies decreased over time. We divided the study period (1994-2019) into three time periods by year of enrollment: 1994-2002 (group one, $n=33$ ), 2003-2010 (group two, $\mathrm{n}=67$ ) and 2011-2019 (group three, $\mathrm{n}=18$ ). We found that median age was 52.0 years (95\% CI: 50.0 , $54.0)$ for group one, 54.0 years $(95 \%$ CI: $53.7,55.9)$ for group two and 59.5 (95\% CI: 56.0, 61.5) for group three (Table 1). In particular, the age disparity in group three $(-7.16$ years, $95 \%$ CI: $-9.38,-4.94)$ was less than group two $(-9.85$ years, $95 \% \mathrm{CI}:-10.79,-8.90, \mathrm{p}=0.071)$ and significantly less then group one $(-12.03$ years, $95 \%$ CI; -14.33, -9.74, $\mathrm{p}=0.008$ ) (Figure 2).

When assessing for sex disparities, we found that female representation on clinical trials was less than expected for the population, but the difference did not reach significance. Mean DPF was -1.23 (95\% CI: - 2.8, 0.34, p = 0.018) compared with the population. However, DPF was not significantly associated with any of study factors and did not change significantly over the study period.

In our study, only $28.8 \%$ (34/118) of trials reported race/ethnic information on ClinicalTrials.gov or publications in PubMed, therefore a detailed analysis could not be performed.

\section{Discussion}

Enrollment of patients on clinical trials should be representative of the patient population, to allow for generalizability of trial results [19]. Enrollment disparities including under-representation of older and female patients is well documented in many types of cancer $[10,11,13,14,20,21]$. We found that age disparities existed in GBM clinical trials with clinical trial participants being a median of 10 years younger than the US GBM population. This is in line with recent work which showed a younger median age of GBM trial participants ( 55.3 years) than the GBM population age (63 years) [22]. The disparities we found were wider than in other types of cancers, including breast ( -7.76 years), prostate (2.66 year), colorectal ( -6.96 year) and lung cancer clinical trials (-8.98 years) [13].

We found that certain types of ICTs were more strongly associated with disparities. Patients enrolled in non-RCT had greater age disparities compared with those in RCT trials. Nonrandomized studies, including Phase I and many Phase II studies, are initiated with limited safety and efficacy data and one can postulate that physicians are less willing to enroll older patients on earlier phase studies or that patients may perceive them as less safe. Nearly half of the trials were funded by industry, similar to a recent report by Cihoric et al. that described the current status for GBM clinical trials [23]. However, we did not find differential representation between industry sponsored or nonindustry sponsored trials. This is in contrast to other work that reported that industry sponsorship of trials is associated with heightened age disparities among trial participants [13].

Sex disparities in clinical oncology research have been documented in the literature in other solid tumors $[10,11,14]$. We found that enrollment of females on GBM clinical trials was 1.2\% lower than expected based on the US female 
GBM population. Recent work found slightly lower rates of enrollment by women [22], but this was less than the discrepancies reported in colorectal and lung cancer (-6.8\%) [14]. Collectively, our data suggest that enrollment of women on GBM clinical trials is largely reflective of the population, keeping in mind that this disease is less common in women.

Our data found a clear decrease in age disparities in GBM clinical trials over a period of almost 25 years which is consistent with trends in many solid tumors [11]. This may be due to the emergence of geriatric oncology as a distinct field, more clinical trials dedicated to older patients and the recognition that placing an upper age limit on eligibility criteria needs to be strongly justified. Additionally, many of the therapies used for the treatment of GBMradiation and temozolomide - are perceived as well tolerated in comparison to many of the cytotoxic therapies used in other solid tumors. However, even in recent years, the age disparity persists, and this has important implications for our understanding of how best to treat older patients. When early phase trials in particular do not represent all patients, we run the risk of developing and approving drugs that may be less tolerable and less efficacious in older patients. It is well described that older patients respond to treatment differently due to drug metabolism, toxicity or biological tumor differences [24-26]. Ultimately, these issues can further contribute to a lack of treatment consensus for older patients with GBM [9] which becomes a public health problem, when nearly half of patients are over the age of 65 [27].

Our study was limited by the data available regarding age, sex and race posted on ClinicalTrials.gov or corresponding publications in PubMed. Data on ClinicalTrials.gov might be registered incorrectly or out-of-date [23,28], and the PubMed database might not capture all relevant clinical trials publications [29] when the time from trial completion to final publication could be over 30 months [28,30]. More than 200 of the included studies have not yet been published and we can only report data on age, sex and race for a fraction of clinical trials. This limitation highlights another reason to call for the standardized reporting of all GBM clinical trials. There was also a lack of information regarding race and ethnicity. This prevented us from exploring these disparities in a meaningful way. As we move forward with the goals of engaging more patients in clinical trials, it is critical that this data be shared in publications to ensure that we are representing all groups and that we are not missing biological differences in tumor response to therapeutics. The ClinicalTrials.gov dataset includes all trials conducted in USA and we are unable to estimate representation on trials in other countries. However, some of these studies were international multicenter studies and the published demographics include patients from other countries. Therefore, our own estimates are not purely representative of the US population.

Our study demonstrated notable age enrollment disparities for GBM clinical trials. This is in the setting of recent efforts by the Society for Neuro-Oncology, the Response Assessment in Neuro-Oncology working group, patient advocacy groups and clinical trial cooperative groups to double clinical trial accrual over the next 5 years [27]. Recently, the US FDA issued a guideline on improving the diversity of patient populations in clinical trials, focusing more on historically under-represented populations including women and older patients [31]. Ongoing efforts are needed in clinical trial design and implementation to ensure equitable participation and that clinical trials are actively recruiting and enrolling participants that represent the entire population. Although research suggests that strict age criteria are less common today in oncology trials, there is increased recognition that strict eligibility criteria regarding performance status, medical comorbidities and laboratory values may preferentially exclude older patients and the need for these criteria should be evaluated strictly in each study [32,33]. We believe our findings are particularly useful for organizations, policy makers, physicians and other stakeholders to better distribute resources, remove enrollment barriers and design inclusive clinical trials in the future so that trial results can be applicable to the true patient population.

\section{Conclusion}

Despite improvement over time, GBM ICTs under-represent older patients. Enrollment by sex on GBM ICT essentially reflects the population. Reporting of race and ethnicity for all ICT should be encouraged. ICTs need to be intentionally designed and implemented to better represent the entire population.

\section{Author contributions}

Y Liu was involved in designing the study, performing statistical analysis and drafting and revising the manuscript. A Wasilewski was involved in designing the study and revising the manuscript. N Mohile was involved in designing the study and revising the manuscript. 
Financial \& competing interests disclosure

The authors have no relevant affiliations or financial involvement with any organization or entity with a financial interest in or financial conflict with the subject matter or materials discussed in the manuscript. This includes employment, consultancies, honoraria, stock ownership or options, expert testimony, grants or patents received or pending, or royalties.

No writing assistance was utilized in the production of this manuscript.

\section{Open access}

This work is licensed under the Attribution-NonCommercial-NoDerivatives 4.0 Unported License. To view a copy of this license, visit http://creativecommons.org/licenses/by-nc-nd/4.0/

\section{Summary points}

- Interventional clinical trials in glioblastoma significantly under-represent older patients.

- Over 25 years, there has been an increase in enrollment of older patients.

- Older patients were less likely to enroll in early phase and nonrandomized trials.

- Although fewer women enrolled on glioblastoma clinical trials, their numbers generally reflect the population.

- There was insufficient data in published trials to determine if there were racial disparities.

- Clinical trials should be designed with eligibility criteria to be inclusive of older patients and their comorbidities.

- Clinical trial data should be published, even if negative, and should report data on race.

\section{References}

Papers of special note have been highlighted as: $\bullet \bullet$ of considerable interest

1. Stupp R, Mason WP, van den Bent MJ et al. Radiotherapy plus concomitant and adjuvant temozolomide for glioblastoma. N. Engl. J. Med. 352(10), 987-996 (2005).

2. Chinot OL, Wick W, Mason W et al. Bevacizumab plus radiotherapy-temozolomide for newly diagnosed glioblastoma. N. Engl. J. Med. 370(8), 709-722 (2014).

3. Stupp R, Taillibert S, Kanner A et al. Effect of tumor-treating fields plus maintenance temozolomide vs maintenance temozolomide alone on survival in patients with glioblastoma: a randomized clinical trial. JAMA 318(23), 2306-2316 (2017).

4. Hurria A, Naylor M, Cohen HJ. Improving the quality of cancer care in an aging population: recommendations from an IOM report. JAMA 310(17), 1795-1796 (2013).

5. Freedman RA, Ruddy KJ. Who are the patients in our clinical trials for cancer? J. Clin. Oncol. 37(18), 1519-1523 (2019).

6. Perry JR, Laperriere N, O'Callaghan CJ et al. Short-course radiation plus temozolomide in elderly patients with glioblastoma. N. Engl. J. Med. 376(11), 1027-1037 (2017).

7. Ostrom QT, Gittleman H, Truitt G, Boscia A, Kruchko C, Barnholtz-Sloan JS. CBTRUS statistical report: primary brain and other central nervous system tumors diagnosed in the United States in 2011-2015. Neuro Oncol. 20(Suppl. 4), iv1-iv86 (2018).

8. Jordan JT, Gerstner ER, Batchelor TT, Cahill DP, Plotkin SR. Glioblastoma care in the elderly. Cancer 122(2), 189-197 (2016).

9. Palmer JD, Bhamidipati D, Mehta $\mathrm{M}$ et al. Treatment recommendations for elderly patients with newly diagnosed glioblastoma lack worldwide consensus. J. Neurooncol. 140(2), 421-426 (2018).

10. Murthy VH, Krumholz HM, Gross CP. Participation in cancer clinical trials: race-, sex-, and age-based disparities. JAMA 291(22), 2720-2726 (2004).

11. Pang HH, Wang X, Stinchcombe TE et al. Enrollment trends and disparity among patients with lung cancer in national clinical trials, 1990 to 2012. J. Clin. Oncol. 34(33), 3992-3999 (2016).

12. Lewis JH, Kilgore ML, Goldman DP et al. Participation of patients 65 years of age or older in cancer clinical trials. J. Clin. Oncol. 21(7), 1383-1389 (2003).

13. Ludmir EB, Mainwaring W, Lin TA et al. Factors associated with age disparities among cancer clinical trial participants. JAMA Oncol. doi:10.1001/jamaoncol.2019.2055 (2019) [Epub ahead of print].

-• Highlights the landscape of age disparities across all cancer clinical trials.

14. Ludmir EB, Fuller CD, Moningi S et al. Sex-based disparities among cancer clinical trial participants. J. Natl Cancer Inst. 112(2), 211-213 (2020).

15. Hess KR, Broglio KR, Bondy ML. Adult glioma incidence trends in the United States, 1977-2000. Cancer 101(10), 2293-2299 (2004).

16. Dolecek TA, Propp JM, Stroup NE, Kruchko C. CBTRUS statistical report: primary brain and central nervous system tumors diagnosed in the United States in 2005-2009. Neuro Oncol. 14(Suppl. 5), v1-v49 (2012). 
17. Hirsch BR, Califf RM, Cheng SK et al. Characteristics of oncology clinical trials: insights from a systematic analysis of ClinicalTrials.gov. JAMA Intern. Med. 173(11), 972-979 (2013).

18. National Cancer Institute. Types of cancer treatment. (2020). www.cancer.gov/about-cancer/treatment/types

19. Unger JM, Cook E, Tai E, Bleyer A. The role of clinical trial participation in cancer research: barriers, evidence, and strategies. Am. Soc. Clin. Oncol. Educ. Book 35, 185-198 (2016).

20. Chang SM, Barker FG, 2nd Schmidt MH et al. Clinical trial participation among patients enrolled in the Glioma Outcomes Project. Cancer 94(10), 2681-2687 (2002).

21. Harrison RA, Anderson MD, Cachia D et al. Clinical trial participation of patients with glioblastoma at The University of Texas MD Anderson Cancer Center. Eur. J. Cancer 112, 83-93 (2019).

22. Ludmir EB, Mandel JJ, McAleer MF, de Groot JF. Disparities along the glioblastoma clinical trials landscape. Neuro Oncol. 21(2), 285-286 (2019).

23. Cihoric N, Tsikkinis A, Minniti G et al. Current status and perspectives of interventional clinical trials for glioblastoma - analysis of ClinicalTrials.gov. Radiat. Oncol. 12(1), 1 (2017).

24. Chen H, Cantor A, Meyer J et al. Can older cancer patients tolerate chemotherapy? A prospective pilot study. Cancer 97(4), 1107-1114 (2003).

25. Mariano C, Francl M, Pope J, Wong L, Lim HJ, Lohrisch C. Comparison of toxicity experienced by older versus younger patients enrolled in breast cancer clinical trials. Clin. Breast Cancer 15(1), 73-79 (2015).

26. Sud S, Lai P, Zhang TH, Clemons M, Wheatley-Price P. Chemotherapy in the oldest old: The feasibility of delivering cytotoxic therapy to patients 80 years old and older. J. Geriatr. Oncol. 6(5), 395-400 (2015).

27. Lee EQ, Chukwueke UN, Hervey-Jumper SL et al. Barriers to accrual and enrollment in brain tumor trials. Neuro Oncol. doi:10.1093/neuonc/noz104 (2019) [Epub ahead of print].

-. A multidisicplinary group highlights the barriers to accural experience by brain tumor patients.

28. Vanderbeek AM, Rahman R, Fell G et al. The clinical trials landscape for glioblastoma: is it adequate to develop new treatments? Neuro Oncol. 20(8), 1034-1043 (2018).

29. Huser V, Cimino JJ. Precision and negative predictive value of links between ClinicalTrials.gov and PubMed. AMIA Annu. Symp. Proc. 2012, 400-408 (2012).

30. Ross JS, Tse T, Zarin DA, Xu H, Zhou L, Krumholz HM. Publication of NIH funded trials registered in ClinicalTrials.gov: cross sectional analysis. BMJ 344, d7292 (2012).

31. U.S. Food \& Drug Administration. Enhancing the diversity of clinical trial populations - eligibility criteria, enrollment practices, and trial designs guidance for industry. (2019). www.fda.gov/regulatory-information/search-fda-guidance-documents/enhancing-diversity-c linical-trial-populations-eligibility-criteria-enrollment-practices-and-trial

32. Ludmir EB, Subbiah IM, Mainwaring W et al. Decreasing incidence of upper age restriction enrollment criteria among cancer clinical trials. J. Geriatr. Oncol. 11(3), 451-454 (2020).

-• Demonstrates the gradual easing of age restrictions in cancer clinical trials.

33. Lee EQ, Weller M, Sul J et al. Optimizing eligibility criteria and clinical trial conduct to enhance clinical trial participation for primary brain tumor patients. Neuro Oncol. doi:10.1093/neuonc/noaa015 (2020) [Epub ahead of print].

-. Describes measure that can be taken to reduce barriers to trial accrual in brain tumor patients by modifying eligibility criteria. 
